\title{
Corrección de ptosis palpebral por la incisión de blefaroplastia
}

\section{Upper eyelid ptosis treatment using blepharoplasty incision}

\author{
Novo Torres, A.*, Salvador Sanz, J .F.**, Lorda Barraguer, E.**, Laredo Ortiz, C.*
}

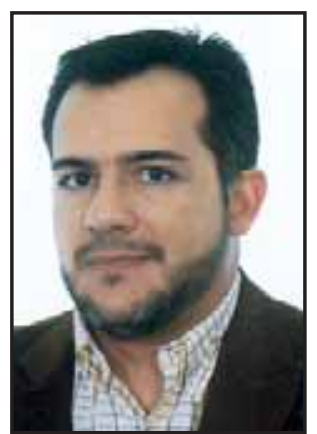

Novo Torres, A.

\section{Resumen}

Se entiende por blefaroptosis el nivel anormalmente bajo del párpado superior durante la mirada directa. Los mejores resultados se obtienen con las técnicas de resección o avance del músculo elevador o su aponeurosis, siempre que el elevador posea una función adecuada. Nuestra intención es presentar una modificación a la técnica de Blaskovics para la corrección de blefaroptosis moderadas, con buena función del elevador

Presentamos una serie de 10 casos donde se utilizó una variante de la operación de Blaskovics de resección del elevador para la corrección quirúrgica de la ptosis palpebral. De los 10 casos, 7 fueron bilaterales y 3 unilaterales. En todos ellos la función del elevador se situaba entre 4 y $8 \mathrm{~mm}$ y el grado de ptosis fue mayor de $4 \mathrm{~mm}$ con disminución del campo visual en la mirada directa. La técnica quirúrgica incluyó un abordaje cutáneo mediante la incisión horizontal de Blefaroplastia superior, la exposición amplia del músculo elevador y su aponeurosis, respetando las bolsas grasas orbiculares y separándolo de la conjuntiva palpebral.

En todos los casos se obtuvo corrección de las ptosis. El grado de asimetría fue inferior a $0,3 \mathrm{~cm}$. Un caso presentó en el postoperatorio inmediato dehiscencia de la sutura en su lado medial y tuvo que ser reintervenido a los 10 días. El edema palpebral postoperatorio se resolvió a partir del $2^{\circ}$ día postoperatorio desapareciendo hacia el día $8^{\circ}$ y permitiendo una valoración fiable en dicha fecha. El grado de satisfacción de los paciente fue bueno o muy bueno y el campo visual fue completo en todos los pacientes

Las técnicas clásicas y sus modificaciones para la corrección de los casos de ptosis siguen teniendo validez. Con esta modificación que respeta la conjuntiva palpebral ocular se evita añadir morbilidad al proceso quirúrgico. Pensamos que la integridad de la conjuntiva redunda en un mayor confort postoperatorio al paciente, disminuye el edema conjuntival y por tanto la recuperación es mas rápida; posibilita también la cirugía de rescate en caso de correcciones insuficientes y evita el problema de sobrecorrección con exceso de resección de tejido conjuntival.

En resumen se trata de una modificación quirúrgica que facilita la técnica, disminuye las complicaciones postoperatorias, mejora la recuperación y evita la sensación de cuerpo extraño intraocular de una resección conjuntival.

Palabras clave Blefaroptosis, Blefaroplastia.

Código numérico 2506, 261
Blepharoptosis is the abnormally low of the top eyelid during the direct look. Best results are obtained by resection or advance of the elevator muscle or aponeurosis, whenever the elevator has a suitable function. We present a modification to the Blaskovics's technique for correction of moderate blepharoptosis, with good function of the elevator muscle.

We present a serie of 10 cases where we used a variant of Blaskovics's technique of resection of the elevator for surgical correction of ptosis. Seven were bilateral cases and 3 unilateral ones. In all the cases the elevator function was between 4 and $8 \mathrm{~mm}$ and ptosis was major than $4 \mathrm{~mm}$ with visual field decrease in the direct look.

Surgical technique included a cutaneous horizontal incision of Blepharoplasty, wide exhibition of elevator muscle and aponeurosis, respecting orbicular fatty bags and separating it from the palpebral conjunctive.

In all the cases correction of the ptosis were obtained. The grade of asymmetry was lower than $0,3 \mathrm{~cm}$. One case presented in the inmediate postoperatory opening of the suture in his medial side and had to be re-taken after 10 days. The palpebral postoperative edema was solved between $2-8$ th day allowing evaluation in that moment. Patient's satisfaction was good or very good and the visual field was complete in all patients

Classic surgical techniques and its modifications for correction of ptosis keep on being valid. With this modification that respects conjuctive we avoid to add morbidity to the surgical process. We think that conjunctive integrity produces a major postoperative comfort to the patient, diminishes the edema and improve the recovery; additionally it makes possible the rescue surgery in case of insufficient correction and avoids over-correction with excess of resection of conjuntive tissue.

In summary is a surgical modification that facilitates the technique, diminishes postoperative complications, improves the postoperative recovery and avoids the sensation of intraocular strange body of the conjuntival resection.

Key words Blepharoptosis, Blepharoplasty.

Código numérico 2506, 261 
Introducción

La ptosis del párpado superior o blefaroptosis es el nivel anormalmente bajo del borde libre del párpado superior durante la mirada directa hacia delante; sugiere que en reposo, el párpado superior interfiere con el campo visual, o por lo menos sobrepasa el limbo corneal. En otras palabras, la caída del párpado sobre el borde superior de la cornea (Fig. 1). Su etiología puede ser congénita o adquirida y esta última puede clasificarse en neurogénica, biogénica, aponeurótica o mecánica y iatrogénica (efecto secundario de la Toxina Botulina) $(1,3)$.

Los mejores resultados funcionales y estéticos se obtienen con la resección o avance del músculo elevador o su aponeurosis, a condición de que el elevador posea función adecuada. Antes de decidir el tipo de intervención indicada, debe establecerse el estado de la función muscular, la posición del párpado respecto al limbo corneal y la ubicación del pliegue palpebral.

A modo de esquema, cuando la función del elevador es superior a $10 \mathrm{~mm}$ y el grado de ptosis es mínimo o inferior a $2 \mathrm{~mm}$, se pueden utilizar, con buenos resultados, la técnica de Fasanella-Servat $(4,5)$ o sus modificaciones. Cuando hay buena función del elevador y el grado de ptosis es mayor de $2 \mathrm{~mm}$ e inferior a $4 \mathrm{~mm}$, se obtienen los mejores resultados si se explora la aponeurosis del elevador para proceder a su avance, plegamiento o reaproximación (cirugía de la aponeurosis). Cuando la función del elevador es débil (4 a $10 \mathrm{~mm}$ ) o escasa $(<4 \mathrm{~mm})$ se utilizan las técnicas de resección del elevador y la suspensión frontal respectivamente $(3,10)$.

La intención del artículo es presentar nuestra experiencia con la técnica de Blaskovics(11) para la corrección de blefaroptosis moderas con buena función del elevador y destacar los beneficios del refinamiento en el abordaje que nosotros realizamos

\section{Material y Método}

Presentamos una serie de 10 casos recogidos en el hospital General Universitario de Alicate y Hospital Internacional Medimar, en los que se utilizó una variante de la técnica de Blaskovics de resección del elevador para la corrección quirúrgica de la ptosis palpebral (11). De los 10 casos, 7 fueron bilaterales y 3 unilaterales. En todos, la función del elevador se situaba entre 4 y $8 \mathrm{~mm}$ y el grado de ptosis era mayor de $4 \mathrm{~mm}$ con disminución del campo visual en la mirada directa.

\section{Técnica quirúrgica}

La técnica incluye el abordaje cutáneo mediante incisión horizontal, como para blefaroplastia, sobre el reborde tarsal del párpado superior y exposición completa del músculo orbicular. Sección de una tira del músculo orbicular para exponer la aponeurosis del elevador (Fig. 2). Disección amplia de la aponeurosis y del músculo elevador en su superficie respetando las bolsas grasas orbiculares (Fig. 3). Identificación de los bordes del músculo elevador y disección de la cara profunda. Una vez liberado el elevador manteniendo la conjuntiva integra (Fig. 4), se clampa con una pinza de hemostasia la aponeurosis y se mide la resección a nivel de su inserción en el tarso (aproximadamente los $\mathrm{mm}$ que se pretenda elevar el párpado).

Se marca con tinta el límite de resección y se procede a la misma (Fig. 5. y 6). Tensión y sutura del borde de resección del músculo al tarso con puntos de vicryl 6/0 consiguiendo una hipercorrección de 1-2 mm. Cierre cutáneo (Fig. 7).

Tabla I. Resumen de los casos. Sexo: Mujeres (M), Varones (V), Edad en años, Derecha (D), Izquierda (I), Anestesia Local (L), Sedación (S) y Anestesia General (G).

Casos Edad Ptosis bilateral Función del Elevador Resección del Elevador Asimetría Anestesia Complicaciones

$\begin{array}{lccccccc}1 \mathrm{M} & 72 & \mathrm{D} & 4 \mathrm{~mm} & 6 \mathrm{~mm} & \text { No } & \text { L+S } & \text { No } \\ 2 \mathrm{M} & 25 & \mathrm{I} & 5 \mathrm{~mm} & 9 \mathrm{~mm} & \text { No } & \text { L+S } & \text { No } \\ 3 \mathrm{M} & 65 & \text { Sí } & 6 \mathrm{~mm} & 4 \mathrm{~mm} & \text { No } & \text { L+S } & \text { No } \\ 4 \mathrm{~V} & 43 & \text { Sí } & 6 \mathrm{~mm} & 5 \mathrm{~mm} \mathrm{D} \mathrm{y} 4 \mathrm{~mm} \mathrm{I} & \text { No } & \text { G } & \text { No } \\ 5 \mathrm{M} & 44 & \text { Sí } & 8 \mathrm{~mm} & \text { Plicatura } & 1 \mathrm{~mm} & \text { L+S } & \text { No } \\ 6 \mathrm{~V} & 61 & \text { Sí } & 6 \mathrm{~mm} & 4 \mathrm{~mm} & 1 \mathrm{~mm} & \text { G } & \text { Deshisencia } \\ 7 \mathrm{M} & 48 & \text { Sí } & 6 \mathrm{~mm} & 6 \mathrm{~mm} \mathrm{D} \mathrm{y} 7 \mathrm{~mm} \mathrm{I} & \text { No } & \text { L+S } & \text { No } \\ 8 \mathrm{~V} & 56 & \text { I } & 4 \mathrm{~mm} & 8 \mathrm{~mm} & \text { No } & \text { L+S } & \text { Conjuntivitis } \\ 9 \mathrm{M} & 55 & \text { Sí } & 7 \mathrm{~mm} & \text { Plicatura } & \text { No } & \text { L+S } & \text { No } \\ 10 \mathrm{M} & 46 & \text { Sí } & 8 \mathrm{~mm} & \text { Plicatura } & 6,7 \mathrm{~mm} & & 2 \\ & 51,5 & & 4-8 \mathrm{~mm} & & & \end{array}$




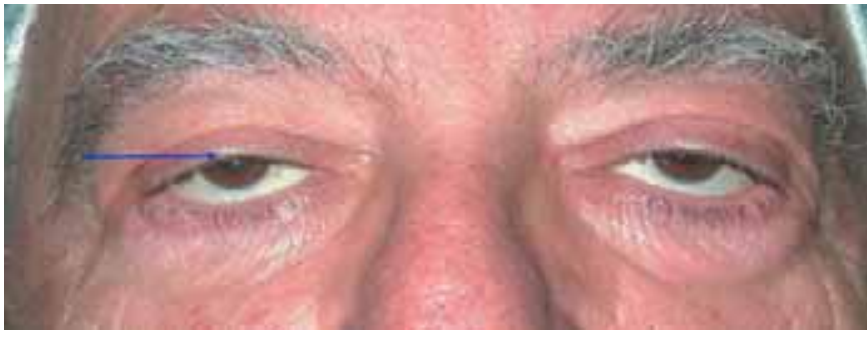

Fig. 1. Paciente con blefaroptosis moderada con aceptable función del elevador. La flecha indica cómo el borde libre del párpado cae sobre la cornea.

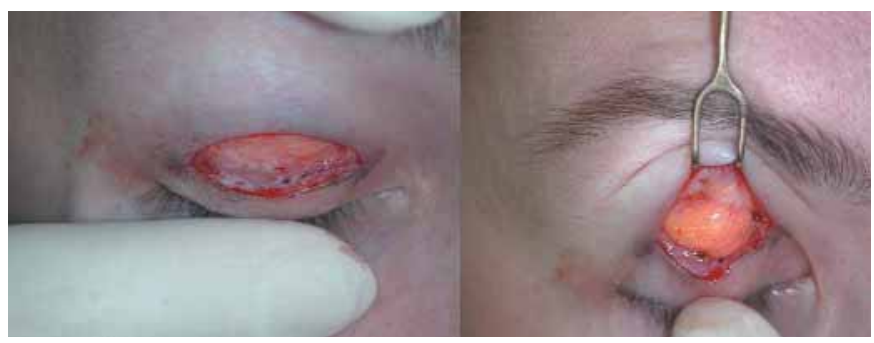

Fig. 3. Exposición de la aponeurosis del elevador para disección amplia en su superficie respetando las bolsas grasas orbiculares.

En los casos de ptosis muy discretas en los que realizamos solo una plicatura de la fascia del elevador sin desinsertarlo, continuamos después con el resto de la blefaroplastia si está indicada.

Presentamos a continuación un resumen de los casos analizados (Tabla I).

\section{Casos Tipo}

Caso 1. Mujer de 72 años que consulta por blefaroptosis unilateral izquierda de causa idiopática, sin antecedentes de interés. Se realiza la corrección unilateral bajo anestesia local más sedación, resección de $6 \mathrm{~mm}$ (Fig. 8).

Caso 2. Mujer de 25 años con síndrome de Bernard Clot Horner secundario, que presentaba una ptosis unilateral derecha. Se realiza la corrección de $9 \mathrm{~mm}$ bajo anestesia local más sedación (Fig. 9).

Caso 3. Mujer de 65 años que consulta para blefaroplastia estética; se realiza corrección bilateral con nuestra modificación más blefaroplastia superior e inferior (Fig. 10).
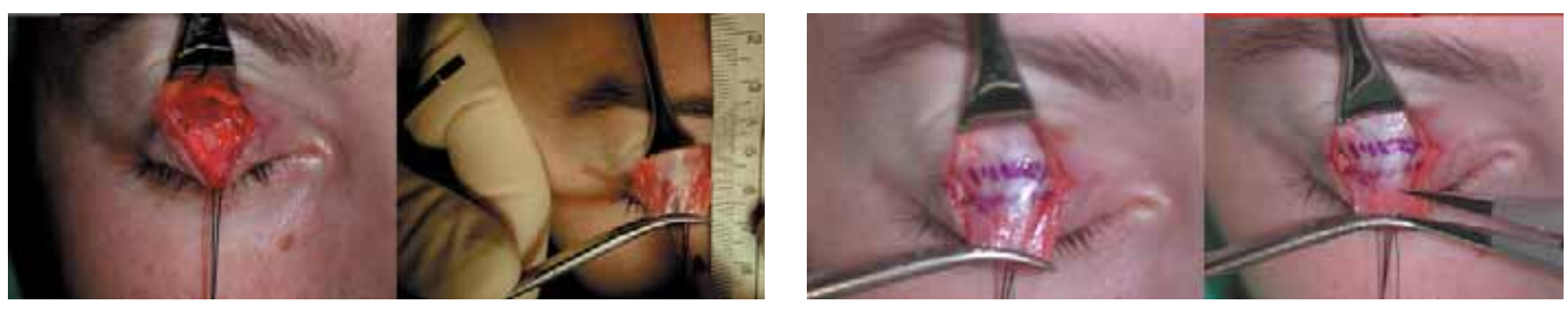

Fig. 5. y 6. Liberalización completa del elevador. Sección de la aponeurosis a nivel de su inserción en el tarso y clampaje con una pinza de hemostasia. Se marca el límite de resección y se procede a la misma.

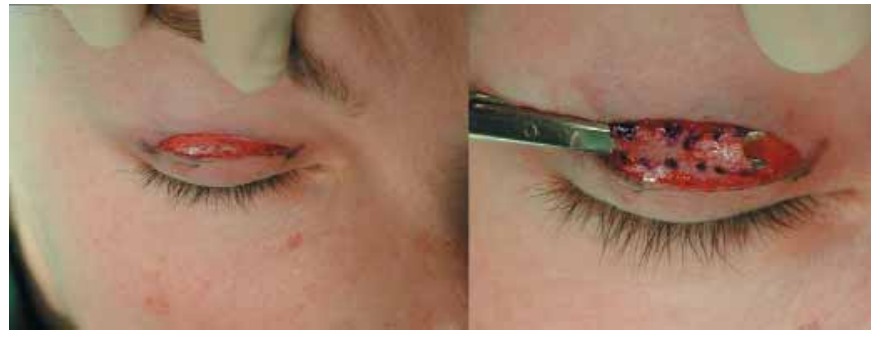

Fig. 2. Técnica quirúrgica abordaje cutáneo mediante incisión horizontal sobre el reborde tarsal del párpado superior que permite una exposición completa del músculo orbicular. Sección de una tira del músculo orbicular.

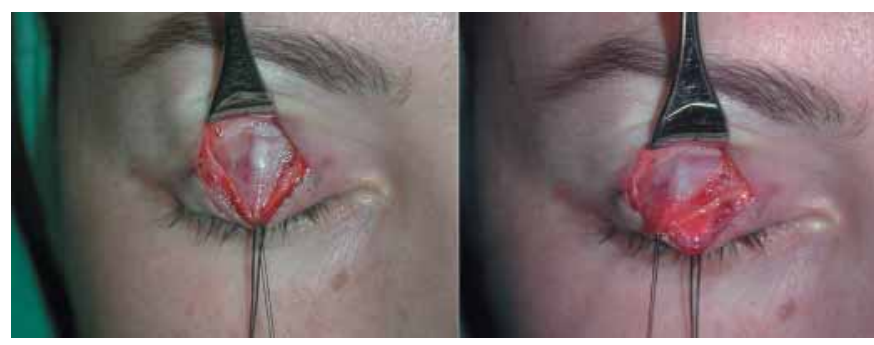

Fig. 4. Identificación de los bordes medial y lateral del músculo elevador, disección de la cara profunda separándola de la conjuntiva palpebral que permanece íntegra.

Caso 4. Varón de 43 años que consulta para rejuvenecimiento facial; se realiza blefaroplastia superior más resección de $5 \mathrm{~mm}$ de la aponeurosis del elevador derecho y $4 \mathrm{~mm}$ del izquierdo (Fig. 11).

Caso 5. Mujer de 44 años que consulta para blefaroplastia; presenta adicionalmente una discreta blefaroptosis. Se realiza blefaroplastia superior con plicatura de la aponeurosis y blefaroplastia inferior transconjutival.

Caso 6. Varón de 61 años que acude a nuestra consulta por blefaroptosis bilateral idiopática. Se realiza bajo anestesia general la corrección de la ptosis. Presenta dehiscencia en el borde medial del ojo izquierdo al $6^{\circ}$ día postoperatorio. Es reintervenido al décimo día, realizando una nueva sutura y limpieza de un pequeño hematoma que podría ser la causa de la dehiscencia. Evoluciona satisfactoriamente.

Caso 7. Mujer de 48 años que acude para blefaroplastia estética; se le hace notar que presenta una blefaroptosis bilateral asimétrica de 6 y $7 \mathrm{~mm}$. Corrección bajo anestesia local más sedación. 


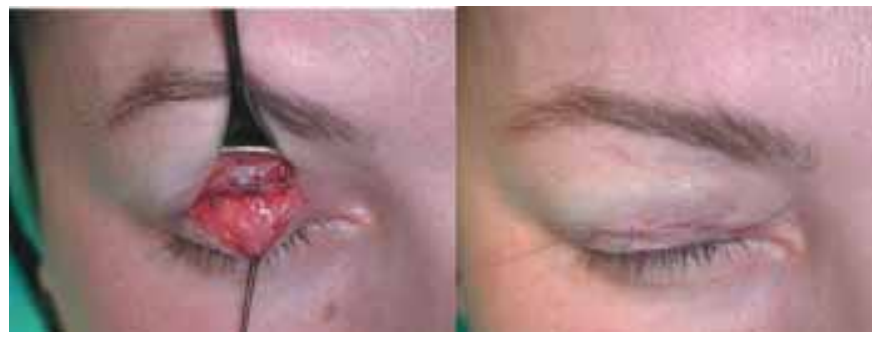

Fig. 7. Sutura del borde de resección del músculo al tarso con puntos de vicryl 6/0 con tensión para conseguir una hipercorrección de 1-2 $\mathrm{mm}$. Cierre cutáneo.

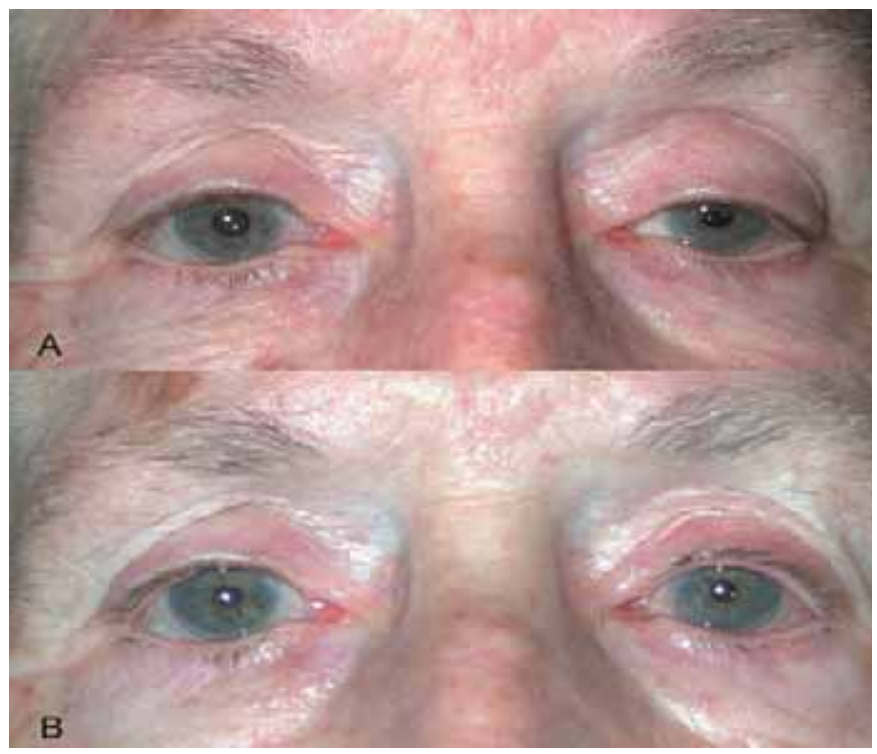

Fig. 8. Caso 1 Corrección unilateral de blefaroptosis izquierda preoperatorio y postoperatorio al mes.

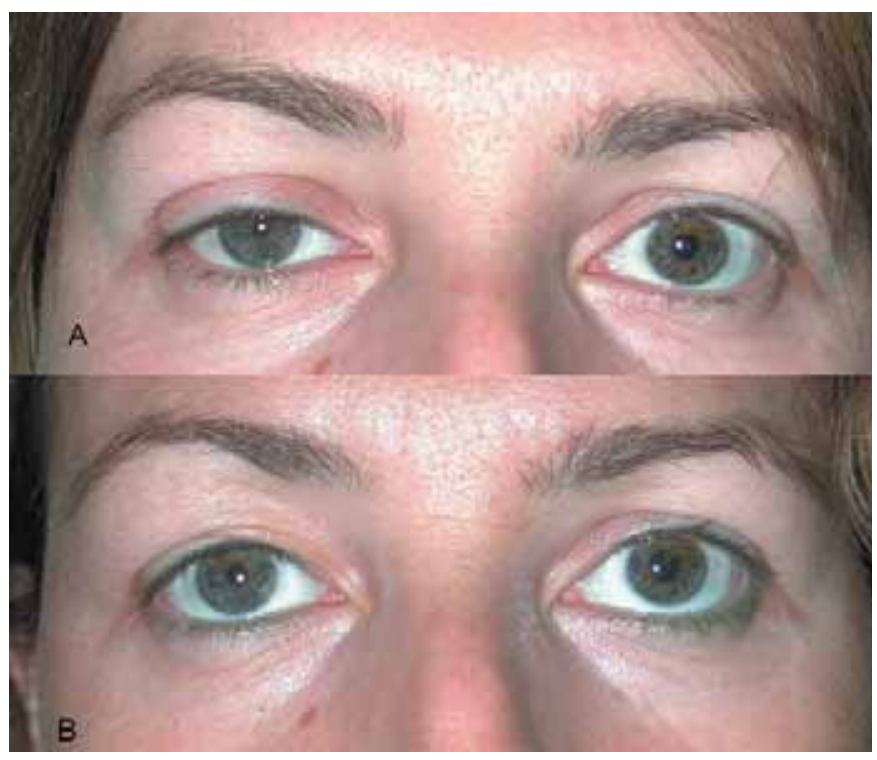

Fig. 9. Caso 2 Síndrome de Bernard Clot Horner. Corrección unilateral de blefaroptosis derecha preoperatorio y postoperatorio al año.

Caso 8. Varón de 56 años con ptosis unilateral izquierda de $8 \mathrm{~mm}$. Corrección con anestesia local

Casos 9 y 10. Mujeres de 55 y 46 años con blefaroptosis bilateral de aproximadamente $5 \mathrm{~mm}$. Se realizó la corrección con la técnica descrita más blefaroplastia superior e inferior.
Resultados

En todos los casos se obtuvo corrección de la ptosis. El grado de asimetría fue inferior a $0,3 \mathrm{~cm}$. Un caso presentó en el postoperatorio inmediato dehiscencia de la sutura en su lado medial y tuvo que ser reintervenido a los 10 días.

La media de resección del elevador fue de $6,7 \mathrm{~mm}$ El edema palpebral postoperatorio se resolvió a partir del $2^{\circ}$ día, desapareciendo hacia el día $8^{\circ}$ y permitiendo una valoración fiable en dicha fecha. El grado de satisfacción de los paciente fue bueno o muy bueno y el campo visual fue completo en todos los pacientes.

En los casos más severos hemos logrado corregir las ptosis sin encontrar asimetrías mayores de $1 \mathrm{~mm}$ y con una correcta función del elevador, por supuesto sin problemas para la oclusión del ojo en ningún caso.

\section{Discusión}

Las técnicas clásicas y sus modificaciones para la corrección de los casos de ptosis siguen teniendo validez $(4,11)$. Nuestra intención al presentar esta modificación, que respeta la conjuntiva palpebral ocular, es evitar añadir morbilidad al proceso quirúrgico. Pensamos que la integridad de la conjuntiva redunda en un mayor confort postoperatorio para el paciente, disminuye el edema conjuntival y por tanto la recuperación es más rápida; adicionalmente posibilita la cirugía de rescate en caso de correcciones insuficientes y evita el grave problema de la sobrecorrección con exceso de resección de tejido conjuntival difícilmente solucionable.

Al realizar la técnica bajo anestesia local más sedación, podemos comprobar con la colaboración del paciente la simetría en los casos unilaterales o asimétricos. Si la resección es correcta (ligeramente hipercorregida $0.5 \mathrm{~mm}$ por encima del limbo), se procede al cierre definitivo. En los casos que se acompañan de una blefaroplastia estética se procede a ella.

$\mathrm{Al}$ disminuir considerablemente el edema al mínimo a partir del $8^{\circ}$ día, se puede valorar el resultado de la corrección y plantear un retoque en caso de asimetría o corrección insuficiente.

Otros autores han propuesto también la utilización de la vía de blefaroplastia para el abordaje de la corrección de la ptosis palpebral; cada una de las técnicas descritas tiene sus ventajas e inconvenientes (12-17). La modificación que nosotros proponemos, en definitiva facilita la corrección de la blefaroptosis, disminuye las complicaciones postoperatorias, mejora la recuperación evitando la sensación de cuerpo extraño intraocular de una resección conjuntival y no aporta ninguna cicatriz al realizarse desde la incisión de blefaroplastia. 


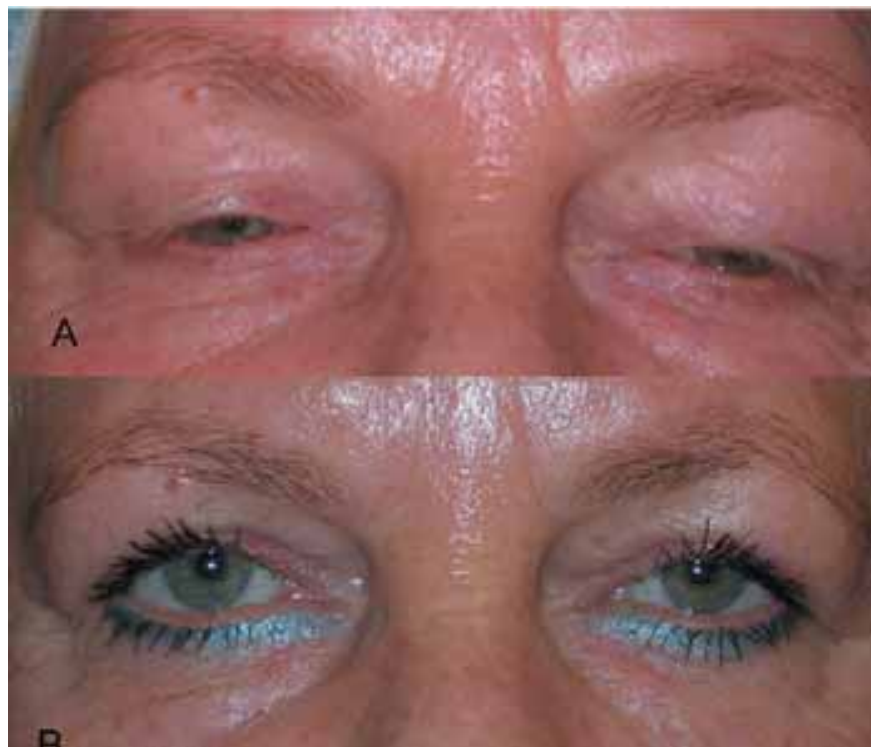

Fig. 10. Caso 3 Blefaroptosis; se realiza corrección bilateral mas blefaroplastia superior e inferior Pre y postoperatorio al año.

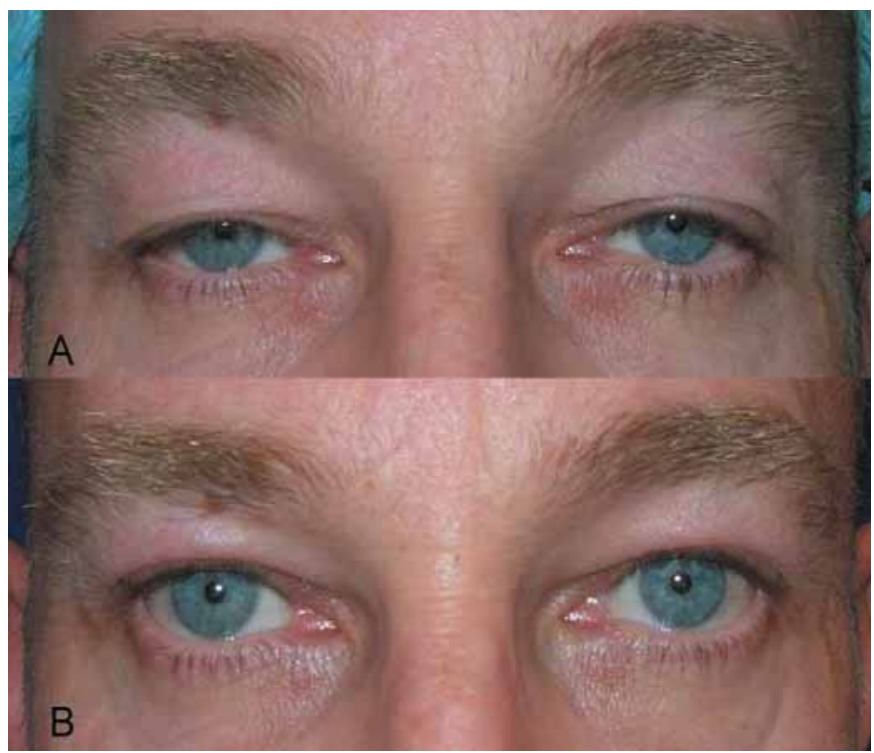

Fig. 11. Caso 4. B lefaroplastia superior mas resección de $5 \mathrm{~mm}$ de la aponeurosis del elevador derecho y de $4 \mathrm{~mm}$ en el izquierdo preoperatori y postoperatorio a los 6 meses.

\section{Conclusiones}

Queremos llamar la atención sobre la incidencia de ptosis moderadas y comentar que con nuestra técnica hemos solucionado los casos más severos de ptosis sin encontrar asimetrías significativas, con correcta función del elevador, y por supuesto sin problemas para la oclusión del ojo, con un postoperatorio más cómodo para el paciente.

Desde que realizamos esta técnica tenemos mayor satisfacción en nuestros pacientes con ptosis y también en aquellos que acuden para blefaroplastia estética cundo presentan ptosis subclínica.
En resumen esta modificación respeta la conjuntiva palpebral ocular, evitando sensación de cuerpo extraño sin añadir morbilidad al proceso quirúrgico. Disminuye el edema conjuntival y por tanto la recuperación del paciente es más rápida, posibilitando la cirugía secundaria de rescate si fuera necesaria.

Dirección del autor

\author{
Dr. Ashley Novo Torres \\ Camino de la Colonia Romana 9- $3^{\circ} \mathrm{C}$ \\ 03016 Alicante. España \\ e-mail: ash_novo@yahoo.com
}

\section{Bibliografía}

1. Finsterer J. "Ptosis: causes, presentation, and management". Aesthetic Plast Surg. 2003;27(3):193.

2. Lepore FE. "Unilateral ptosis and Hering's law". Neurology. 1988;38:319.

3. Putnam, J. R., Nunery, W. R., Tanenbaum, M., and Mc- Cord, C. D., Jr. "Blepharoptosis". In D. D. McCord, M.Tanenbaum, and W. R. Nunery, Oculoplastic Surgery, 3rd Ed. New York: Raven, 1995. Pp. 175-220.

4. Fasanella, R. M., and Servat, J. "Levator resection for minimal ptosis: Another simplified operation”. Arch. Ophthalmol.1961; 65: 493.

5. Fasanella RM. "Surgery for minimal ptosis: the Fasanella-Servant operation, 1973." Trans Ophthalmol Soc UK. 1973;93:425.

6. Jelks GW., Smith BC.: "Reconstrucción de los párpados y de las estructuras asociadas: ptosis del párpado superior". En: Cirugía Plástica. La cara. Tomo 3. McCarthy J.G. Plastic Surgery, Editorial Panamericana. Buenos Aires (Argentina) 1992. Pp: 874-879.

7. Jones, L. T., Quickert, M. H., and Wobig, J. L. "The cure of ptosis by aponeurotic repair". Arch. Ophthalmol. 1975, 93: 629.

8. Fan J. "Frontalis suspension technique with a temporal-fasciaecomplex sheet for repairing blepharoptosis". Aesthetic Plast Surg. 2001;25(3):147.

9. Putterman AM, Urist MJ. "Mueller muscle-conjunctiva resection". Arch Ophthalmol. 1975;93:619.

10. Holmstrom H, Santanelli F. "Suspension of the eyelid to the check ligament of the superior fornix for congenital blepharoptosis". Scand J Plast Reconstr Surg Hand Surg. 2002;36(3):149.

11. Blaskovics L: "New operation for ptosis with shortening of the levator and tarsus". Arch Ophthalmol 1923, 52:563.

12. Baroody M; Holds J. B. Sakamoto D. K. MD; Vick V.L; Hartstein M. E. "Small Incision Transcutaneous Levator Aponeurotic Repair for Blepharoptosis". Annals of Plastic Surgery. 2004, 52(6):558

13. Jones LT, Quickert MH, Wobig JL. "The cure of ptosis by aponeurotic repair." Arch Ophthalmol. 1975;93:629.

14. Anderson RL, Beard C. "The levator aponeurosis: attachments and their clinical significance". Arch Ophthalmol. 1977;95:1437.

15. McCord CD. "An external minimal ptosis procedure: external tarsoaponeurectomy". Trans Am Acad Ophthalmol Otolaryngol. 1975;79:683.

16. Cook BE, Lemke BN, Lucarelli MJ. "Small-incision cosmetic surgical techniques: small-incision external levator repair alone and combined with upper eyelid blepharoplasty". In: Hopping S, ed. Operative Techniques in Oculoplastic, Orbital, and Reconstructive Surgery. Vol. 3. Philadelphia, PA: WB Saunders Co; 2000.

17. Ramirez OM., Peña G, "Frontalis Muscle Advancement: A Dynamic Structure for the Treatment of Severe Congenital Eyelid Ptosis”. Plast. Reconstr. Surg. 2004 113:1841. 


\section{Comentario al trabajo uCorrección de ptosis palpebral por la incisión de blefaroplastian}

\section{Dr. J. E. Lagarón Comba \\ Cirujano Plástico \\ Hospital 12 de Octubre, Madrid (España)}

Ante todo felicitar a los autores por el trabajo presentado y sus resultados y sobre todo por contribuir a mantener en el seno de la especialidad una parcela, la oculoplástica, como tantas otras amenazada por el creciente interés de otros especialistas que comparten la misma área de actuación.

No obstante quisiera hacer algunas observaciones. Los autores nos presentan 10 casos, cuya motivación y demanda del paciente es, en la mayor parte de los casos, esencialmente estética, y en los que tras su valoración se ha apreciado un cierto componente de ptosis uni o bilateral, cuya causa anatómica parece estar más en relación con relajación aponeurótica del músculo elevador, asociado al efecto mecánico de blefarochalasia, que con una distrofia muscular primaria.

En tales casos, en nuestra opinión, resulta más acertada, la plicatura de la aponeurosis, como de hecho así lo hicieron en tres de los pacientes, que la propia resección del músculo elevador (técnica inicialmente descrita por von Blaskovics). Esto lo demuestra el hecho de haber obtenido resultados muy satisfactorios a pesar de una resección más que moderada del músculo elevador, para ptosis superiores a $4 \mathrm{~mm}$ (el efecto es más próximo a la plicatura que a la resección muscular). Pensamos que para ptosis por distrofia del elevador, el problema no se corrige con éxito con resecciones inferiores a $4 \mathrm{~mm}$. por $\mathrm{mm}$. de ptosis.

Compartimos con los autores las ventajas del abordaje anterior a través de la incisión de blefaroplastia, tanto más cuanto que es esencial readaptar el excedente cutáneo y definir el surco palpebral. Esta modificación a la técnica de Von Blaskovics, que inicialmente se presentó por abordaje posterior (1923) ya había sido sugerida por Everbush y posteriormente modificada por Byron Smith (1967), aplicada como complemento de la blefaroplastia por Carraway (2003) y seguida por otros muchos autores.

\section{Respuesta al comentario del Dr. J.E. Lagarón Comba}

\section{Dr. Ashley Novo Torres}

En primer lugar agradecer los comentarios del Dr. Lagaron Comba y destacar la relevancia de sus observaciones las cuales en su mayoría comparto. Estoy de acuerdo con él en que para mantener este campo abierto en nuestra especialidad debemos diagnosticar y tratar la patología palpebral en todos los aspectos.

En efecto se tratan de casos con buena función del elevador y en su mayor parte blefarochalasias, lo que algunos autores denominan "ptosis senil", de lo contrario utilizaríamos técnicas de suspensión anclada al músculo frontal. Respecto a realizar únicamente una plicatura del elevador, creemos que está indicada solo en los casos de ptosis muy discretas, ya que la resección permite una corrección más ajustada. Con la plicatura se podrían corregir los casos moderados, pero con el inconveniente de dejar un tejido redundante entre la sutura de la plicatura, que si bien es cierto que posteriormente se readapta, es notorio un abultamiento en el postoperatorio precoz; así ha ocurrido en los casos que realizamos solo una plicatura de la fascia del elevador sin desinsertarlo. Dado que realizar el despegamiento de la cara posterior del músculo elevador no aumenta considerablemente el edema al respetar la conjuntiva y el aumento del tiempo quirúrgico es mínimo, vale la pena realizar la desinserción del elevador de la placa tarsal, resección, sutura y luego continuar con el resto de la blefaroplastia si está indicada.

No puedo hacer ningún comentario sobre las modificaciones de la técnica Von Blaskovics por los autores Everbush y Byron Smith ya que no me ha sido posible encontrar referencias bibliográficas. Respecto al artículo de Carraway "Levator advancement technique for eyelid ptosis", Plast Reconstr Surg. 1986; 77(3): 394, es una técnica similar a la que nosotros realizamos, matizando que en ningún momento sugerimos que la brillante idea de corregir la ptosis palpebral desde la incisión de blefaroplastia sea una técnica original nuestra.

Al igual que el Dr. Lagaron Comba quiero insistir en las ventajas del abordaje anterior, a través de la incisión de blefaroplastia, tanto más cuanto que es esencial readaptar el excedente cutáneo y definir el surco palpebral. Finalmente, destacar el cómodo postoperatorio para el paciente. 